\title{
Quercetin protects against high glucose-induced damage in bone marrow-derived endothelial progenitor cells
}

\author{
LI-RONG ZHAO ${ }^{*}$, YU-JUN DU ${ }^{2 *}$, LEI CHEN ${ }^{3}$, ZHI-GANG LIU ${ }^{3}$, \\ YUE-HAI PAN ${ }^{3}$, JIAN-FENG LIU ${ }^{3}$ and BIN LIU ${ }^{3}$ \\ Departments of ${ }^{1}$ Ultrasound, ${ }^{2}$ Nephrology, ${ }^{3}$ Hand Surgery, \\ The First Hospital of Jilin University, Changchun, Jilin 130021, P.R. China
}

Received January 31, 2014; Accepted July 4, 2014

DOI: $10.3892 / \mathrm{ijmm} .2014 .1852$

\begin{abstract}
Endothelial progenitor cells (EPCs), a group of bone marrow-derived pro-angiogenic cells, contribute to vascular repair after damage. EPC dysfunction exists in diabetes and results in poor wound healing in diabetic patients with trauma or surgery. The aim of the present study was to determine the effect of quercetin, a natural flavonoid on high glucose-induced damage in EPCs. Treatment with high glucose $(40 \mathrm{mM})$ decreased cell viability and migration, and increased oxidant stress, as was evidenced by the elevated levels of reactive oxygen species (ROS), malondialdehyde (MDA) and superoxide dismutase in bone marrow-derived EPCs. Moreover, high glucose reduced the levels of endothelial nitric oxide synthase (eNOS) phosphorylation, nitric oxide (NO) production and intracellular cyclic guanosine monophosphate (cGMP). Quercetin supplement protected against high glucose-induced impairment in cell viability, migration, oxidant stress, eNOS phosphorylation, NO production and cGMP levels. Quercetin also increased Sirt1 expression in EPCs. Inhibition of Sirtl by a chemical antagonist sirtinol abolished the protective effect of quercetin on eNOS phosphorylation, NO production and cGMP levels following high glucose stress. To the best of our knowledge, the results provide the first evidence that quercetin protects against high glucose-induced damage by inducing Sirt1-dependent eNOS upregulation in EPCs, and suggest that quercetin is a promising therapeutic agent for diabetic patients undergoing surgery or other invasive procedures.
\end{abstract}

\section{Introduction}

Vascular endothelial cells (ECs) are a dynamic border between circulating blood and the surrounding tissue. If ECs were

Correspondence to: Dr Bin Liu, Department of Hand Surgery, The First Hospital of Jilin University, 71 Xinmin Street, Changchun, Jilin 130021, P.R. China

E-mail: liub09@126.com

*Contributed equally

Key words: quercetin, endothelial progenitor cells, Sirt1, eNOS, high glucose impaired, endothelial progenitor cells (EPCs), a group of bone marrow-derived cells identified in 1997 (1), would contribute to postnatal vasculogenesis (2). Generally, EPCs are mobilized from the bone marrow into peripheral blood and are home to damaged areas for neo-angiogenesis (2). EPC dysfunction is known to be a critical event of diabetes (3). Circulating EPCs are reduced in peripheral vascular complications of type 1 and 2 diabetes mellitus (4-5). Many factors involved in the pathophysiology of diabetes, such as hyperglycemia, lead to EPC dysfunctions. In vitro studies also demonstrated that high glucose resulted in cellular injury in EPCs by inducing oxidant stress (6), apoptosis (7) and the downregulation of endothelial nitric oxide synthase (eNOS) (8). Since EPC dysfunction may result in poor wound healing in diabetic patients with trauma or surgery, restoring the biological functions of EPCs for these patients is crucial.

Flavonoids have recently received much attention as potential preventive and therapeutic agents for diabetes (9-10). Quercetin, a ubiquitous flavonol abundantly found in plant products such as capers, lovage, apples, onions and grapes, possesses antioxidative and anti-inflammatory properties (11). Recently, the protective and therapeutic value of quercetin in diabetes has been recognized. Oral administration of quercetin in rats with type 1 diabetes significantly decreased incremental plasma glucose levels (12). Quercetin had beneficial effects on cell membranes altered in diabetic conditions, by restoring transmembrane potential (13). Moreover, quercetin protected against diabetes-induced exaggerated vasoconstriction, adventitial leukocyte infiltration, endothelial pyknosis and increased collagen deposition in rats (14). Thus, the ability of quercetin to protect against the deleterious effects of hyperglycemia and to improve glucose metabolism and intake must be considered as it affects treatment strategy. Notably, quercetin has been tested in a double-blind randomized controlled clinical trial (15). In women with type 2 diabetes, quercetin reduced systolic blood pressure, serum concentration of tumor necrosis factor- $\alpha$ (TNF- $\alpha)$ and interleukin-6 (IL-6) (15).

Based on the fact that quercetin has significant beneficial effects for diabetes, we hypothesized that there are likely more molecular mechanisms underlying the protective effects of quercetin in diabetes. In this study, we investigated the effect of quercetin on high glucose-induced damage in EPCs. To the 
best of our knowledge, the results provided the first evidence that quercetin protects against high glucose-induced damage by inducing Sirt1-dependent eNOS upregulation in EPCs.

\section{Materials and methods}

Reagents. Quercetin (catalogue no: Q4951) and antibodies against phosphor-eNOS (p-eNOS), and total-eNOS were purchased from Cell Signaling Biotechnology (Danvers, MA, USA). The antibody against Sirt1 and the Sirt1 inhibitor sirtinol, was purchased from Santa Cruz Biotechnology, Inc. (Santa Cruz, CA, USA). The antibody against $\beta$-actin was purchased from Sigma (St. Louis, MO, USA). The cell viability assay [cell counting kit-8 (CCK-8)] was purchased from Dojindo Molecular Technologies, Inc. (Kumamoto, Japan). Dichlorodihydrofluorescein diacetate (DCFH-DA) was purchased from Invitrogen Life Technologies (Carslbad, CA, USA). Nitric oxide (NO) assay was purchased from Abcam (Cambridge, MA, USA). Malondialdehyde (MDA) and cyclic guanosine monophosphate (cGMP) ELISA kits were purchased from Cell Biolab, Inc. (San Diego, CA, USA).

Bone marrow cell isolation and culture. Bone marrow-derived EPCs were prepared as previously described (16). Bone marrow was harvested from the femurs and tibiae of 2-month-old male C57BL/6J mice (Changchun Institute of Biological Products, Changchun, China). The mononuclear cell fraction was isolated with Ficoll (Amersham Pharmacia Biotech, Inc., Piscataway, NJ, USA). Cells were seeded in fibronectin-coated six-well plates (Corning, Lowell, MA, USA) and cultured in Dulbecco's modified Eagle's medium (DMEM) supplemented with a specific mixture of vascular endothelial growth factor, fibroblast growth factor-2, insulin-like growth factor-1, epidermal growth factor, hydrocortisone, gentamicin, amphotericin-B, $5 \%$ fetal bovine serum and ascorbic acid (EGM2-MV; Lonza, Walkersville, MD, USA). The concentration of glucose in this medium was $5.5 \mathrm{mM}$. To produce a high glucose challenge in EPCs, the EPCs were cultured in high glucose $(40 \mathrm{mM})$.

Cell viability assay. Viability of EPCs was evaluated using a non-radioactive CCK-8 assay as previously described (17-18). High glucose incubated EPCs were treated with quercetin ( 20 or $100 \mu \mathrm{M}$ ) for $48 \mathrm{~h}$. The control cells were cultured in normal glucose medium. After discarding the medium, the cells were incubated with $10 \mu \mathrm{l}$ of CCK-8 solution for $3 \mathrm{~h}$ at $37^{\circ} \mathrm{C}$. The optical density at $450 \mathrm{~nm}$ was analyzed in a microplate reader (Thermo Fisher Scientific Inc., Waltham, MA USA). Experiments were performed in duplicate.

Transwell migration assay. EPCs $\left(1 \times 10^{5}\right)$ were placed in the upper chamber of a 24-well transwell migration insert (pore size, $5 \mu \mathrm{m}$ ) with DMEM in the presence or absence of high glucose and quercetin (20 and $100 \mu \mathrm{M}$, respectively). The lower chamber contained migration medium (EBM-2, 0.5\% BSA, $10 \% \mathrm{FBS}, 100 \mathrm{IU} / \mathrm{ml}$ penicillin, and $100 \mu \mathrm{g} / \mathrm{ml}$ streptomycin) (19). After migration for $24 \mathrm{~h}$, the non-migrating cells on the upper side of the membrane were wiped away, while the cells on the lower side of the membrane were fixed, excised, mounted with DAPI containing mounting medium and counted on a fluorescent microscope (IX71; Olympus, Tokyo, Japan).
Reactive oxygen species (ROS) assay. Intracellular ROS production was evaluated by incubating the cells with $30 \mu \mathrm{M}$ $\mathrm{DCFH}-\mathrm{DA}$ at $37^{\circ} \mathrm{C}$ for $1 \mathrm{~h}$. The fluorescence was revealed by fluorescence microscopy, using the same exposure conditions in each experiment (20). The cells were counted by two blinded readers obtaining similar results. The DCFH-DA fluorescence was calculated using Image Pro Plus software (Media Cybernetics Inc., Silver Spring, MD, USA).

Superoxide dismutase and MDA activity assays. Intracellular superoxide dismutase and MDA assays were performed as previously described (21-23). EPCs were treated with high glucose or high glucose plus quercetin $(100 \mu \mathrm{M})$ for $6 \mathrm{~h}$ and lysed using RIPA buffer. The superoxide dismutase level and MDA concentration of each sample were detected according to the manufacturer's instructions using a microplate reader.

Western blotting. Western blotting was performed as previously described (24-25). Briefly, cells were lysed in lysis buffer $(50 \mathrm{mM}$ Tris, pH 7.4, $150 \mathrm{mM}$ sodium chloride, $1 \%$ Nonidet P-40, 1 mM EDTA, $1 \mathrm{mM}$ sodium orthovanate, $1 \mathrm{mM}$ sodium fluoride, $1 \mathrm{mg} / \mathrm{ml}$ leupeptin, $1 \mathrm{mg} / \mathrm{ml}$ aprotinin, $1 \mathrm{mg} / \mathrm{ml}$ pepstatin $\mathrm{A}$, and $1 \mathrm{mM}$ PMSF) (26). Cell lysates were centrifuged at $10,000 \mathrm{x} \mathrm{g}$ for $10 \mathrm{~min}$ and the supernatants were collected. The samples were boiled for $10 \mathrm{~min}$. The protein concentration was determined using a BCA assay. Equal amounts of protein were separated by $10 \%$ SDS-PAGE and transferred to nitrocellulose by standard procedures (27). Membranes were incubated overnight at $4^{\circ} \mathrm{C}$ with antibodies against p-eNOS (1:200), t-eNOS $(1: 500)$, Sirt1 $(1: 250)$ and $\beta$-actin $(1: 1,000)$. After incubation with the corresponding secondary antibodies $(1: 5,000)$, the membranes were washed three times and bands were detected by the enhanced chemoluminescence kit (ECL; Amersham Pharmacia Biotech, Inc.) (28).

NO production measurement. NO concentrations in EPCs were determined by detecting the concentration of nitrite, the stable product of NO. EPCs were seeded in 24-well plates at a density of $1 \times 10^{5}$ cells/well. Following treatment with glucose or glucose + quercetin or glucose + quercetin + sirtinol for $12 \mathrm{~h}$, the medium was collected and NO content in the medium (NO production) was measured using a microplate procedure based on the Griess reaction by a NO assay kit. The optical densities at a wavelength of $560 \mathrm{~nm}$ were obtained using a microplate reader and concentrations of $\mathrm{NO}$ were calculated according to the calibration curve.

Intracellular cGMP measurement. EPCs were treated with glucose or glucose + quercetin or glucose + quercetin + sirtinol for $12 \mathrm{~h}$. The cells were then lysed using a RIPA buffer. The protein concentration was determined by a BCA assay. The cGMP level was measured using a commercial cGMP ELISA kit according to the manufacturer's instructions.

Statistical analysis. Statistical calculations were performed using the GraphPad Prism 5 software program (GraphPad Software, Inc., La Jolla, CA, USA). The data are expressed as the means \pm SEM and compared using ANOVA with Tukey correction. Statistical significance was set at $\mathrm{P}<0.05$. 


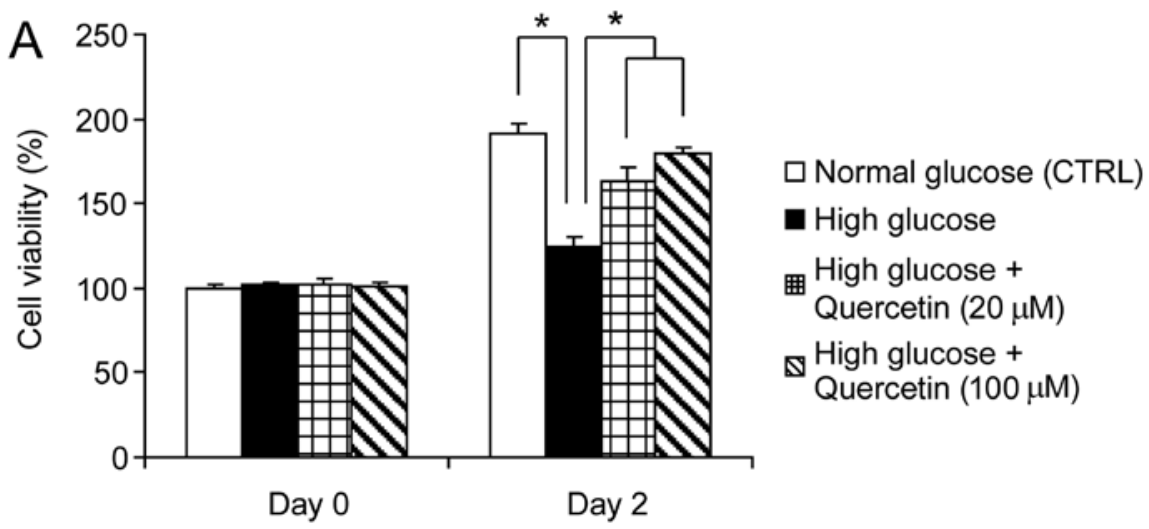

B

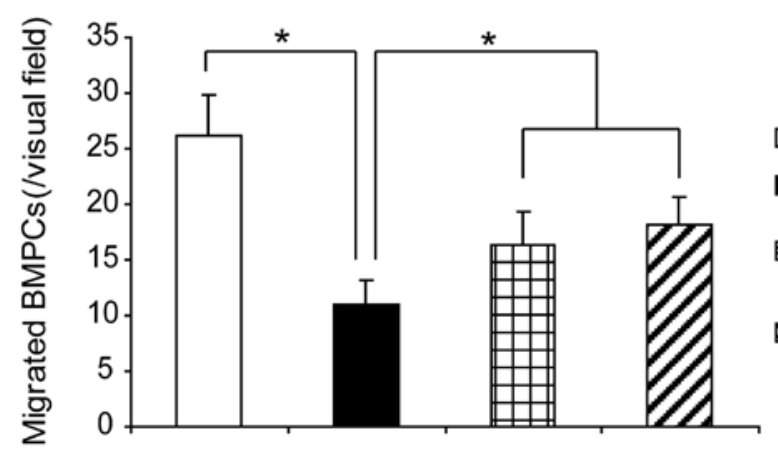

$\square$ Normal glucose (CTRL)

High glucose

High glucose +

Quercetin $(20 \mu \mathrm{M})$

${ }^{\text {High glucose }+}$

Quercetin $(100 \mu \mathrm{M})$

Figure 1. Quercetin protects against high glucose-induced injury in endothelial progenitor cells (EPCs). (A) Effects of high glucose and quercetin on the cell viability of EPCs. PCs were treated with the medium of normal glucose $(5.5 \mathrm{mM})$ or high glucose $(40 \mathrm{mM})$ plus quercetin $(20$ and $100 \mu \mathrm{M})$ for 2 days and the cell viability was measured; " $\mathrm{P}<0.05, \mathrm{n}=8$. (B) Effects of high glucose and quercetin on the migration of EPCs. EPCs $\left(1 \times 10^{5}\right)$ were placed in the upper chamber of a 24-well transwell migration insert with Dulbecco's modified Eagle's medium (DMEM) in normal glucose (5.5 mM) or high glucose (40 mM) and quercetin $(20$ and $100 \mu \mathrm{M})$. The lower chamber contained migration medium to induce EPC migration for $24 \mathrm{~h}$; $\mathrm{P}<0.05, \mathrm{n}=8$.

\section{Results}

Quercetin protects against high glucose-induced damage in $E P C s$. First, we studied whether quercetin exerted protective effects on EPCs. As shown in Fig. 1A, high glucose significantly decreased the cell viability at Day 2 in culture EPCs. The two concentrations of quercetin (20 and $100 \mu \mathrm{M})$ attenuated this detrimental effect of high glucose. The effect of quercetin on the cell migration of EPCs was also investigated. Quercetin (20 and $100 \mu \mathrm{M})$ rescued the migration of EPCs impaired by high glucose (Fig. 1B). These results clearly suggested that quercetin protects against high glucose-induced damage in EPCs.

Quercetin decreases high glucose-induced oxidant stress in EPCs. The effects of quercetin on high glucose-induced damage oxidant stress in EPCs were examined. Results of the DCFH-DA assay revealed that high glucose treatment triggered fluorescence in EPCs, suggesting that the ROS level was markedly increased by high glucose (Fig. 2A). Quercetin supplement $(100 \mu \mathrm{M})$ significantly decreased DCFH-DA fluorescence (Fig. 2A). MDA level is an index of endogenous lipid peroxidation. Notably, high glucose also enhanced the MDA level in EPCs (Fig. 2B). Quercetin successfully inhibited the increase of MDA level (Fig. 2B). Total superoxide dismutase levels, major scavengers of ROS, were also determined. High glucose treatment decreased the superoxide level in EPCs, which was partly rescued by quercetin (Fig. 2C). These results suggested that quercetin decreased high glucose-induced oxidant stress in EPCs.

Quercetin prevents high glucose-induced inhibition of eNOS phosphorylation and NO production in EPCs. NO is, not only the key endothelium-derived relaxing factor that plays pivotal roles in maintaining vascular function, but also a potent positive regulator of EPCs (29-30). Thus, we studied the effects of quercetin on NO production in EPCs following high glucose stress. First, we measured eNOS expression, the major enzyme responsible for NO production in EPCs. High glucose did not alter the eNOS expression (Fig. 3A). However, high glucose decreased eNOS phosphorylation, which is critical for eNOS enzymatic activity (Fig. 3A). Quercetin partly inhibited the high glucose-induced decrease of eNOS phosphorylation in EPCs (Fig. 3A). Moreover, we determined NO production in the medium of cultured EPCs. High glucose treatment significantly reduced the NO production of EPCs (Fig. 3B), which was partly blocked by the quercetin supplement (Fig. 3B). To identify the underlying molecular mechanisms, we assayed intracellular cGMP, which is produced by soluble guanylyl cyclases in responding to NO. High glucose decreased the intracellular cGMP level, which was prevented by quercetin (Fig. 3C). These results emphasized that quercetin 
A
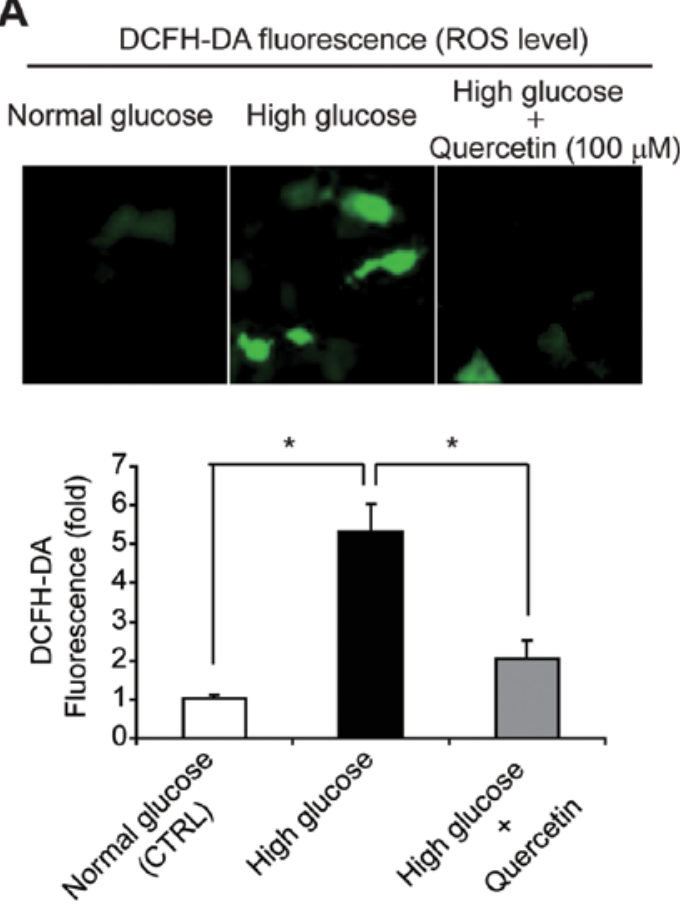

B
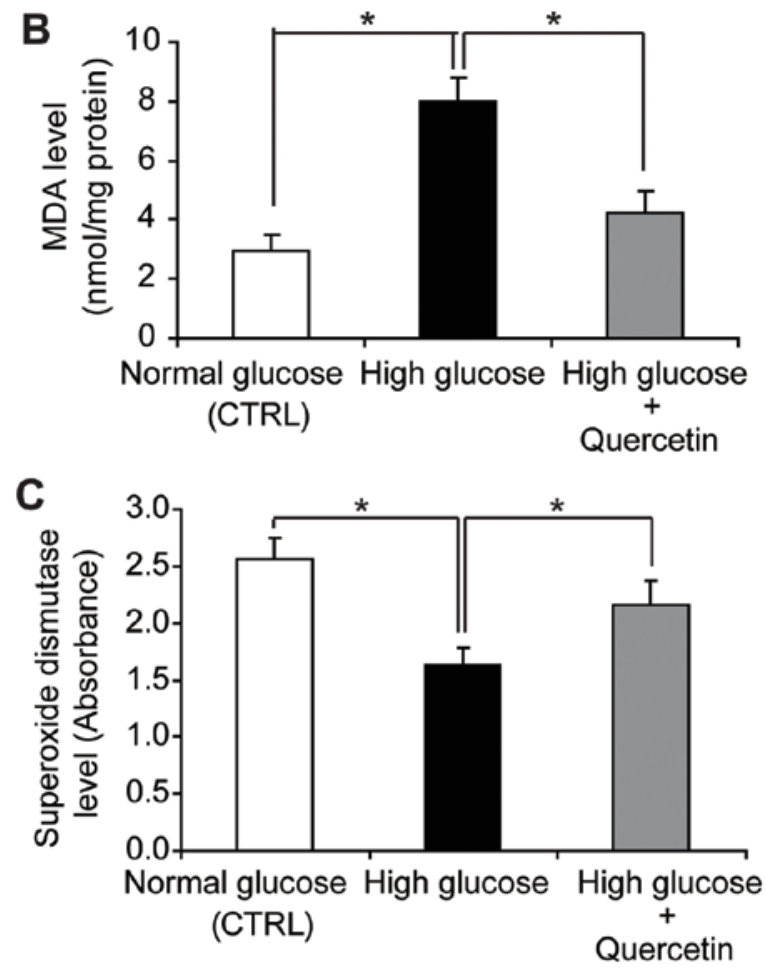

Figure 2. Quercetin decreases high glucose-induced oxidant stress in endothelial progenitor cells (EPCs). (A) Representative images and quantitative analysis of dichloro-dihydro-fluorescein diacetate (DCFH-DA) fluorescence in EPCs treated with high glucose $(40 \mathrm{mM})$ and high glucose + quercetin $(100 \mu \mathrm{M})$ for $24 \mathrm{~h}$; ${ }^{*} \mathrm{P}<0.05, \mathrm{n}=6$. (B) Intracellular malondialdehyde (MDA) levels in EPCs treated with high glucose $(40 \mathrm{mM})$ and high glucose + quercetin $(100 \mu \mathrm{M})$ for $24 \mathrm{~h}$; $\mathrm{P}<0.05$, $\mathrm{n}=6$. (C) Intracellular superoxide dismutase levels in EPCs treated with high glucose $(40 \mathrm{mM})$ and high glucose + quercetin $(100 \mu \mathrm{M})$ for $24 \mathrm{~h}$; "P<0.05, $\mathrm{n}=6$.

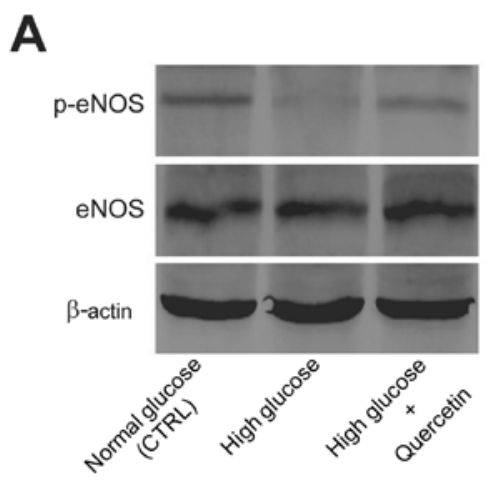

B

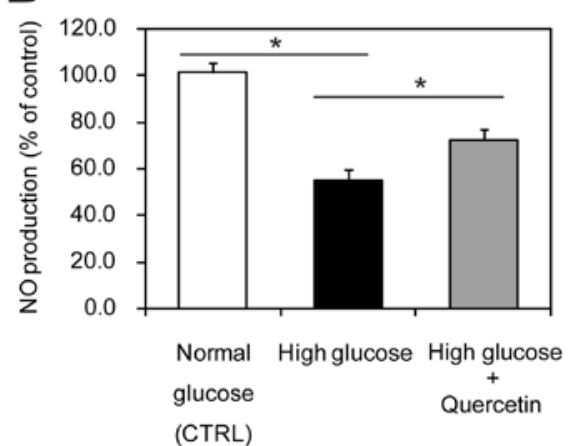

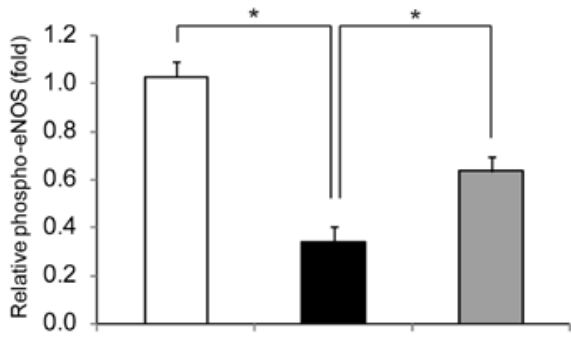

Normal glucose High glucose High glucose

(CTRL) Quercetin

C

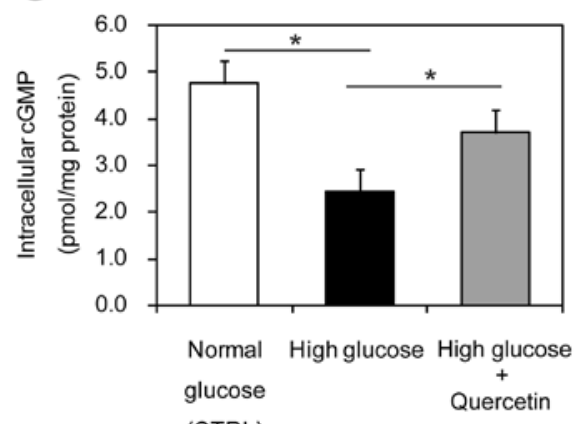

(CTRL)

Figure 3. Quercetin prevents high glucose-induced inhibition of endothelial nitric oxide synthase (eNOS) phosphorylation and nitric oxide (NO) production in endothelial progenitor cells (EPCs). (A) Representative images and quantitative analysis of eNOS phosphorylation in EPCs treated with high glucose (40 mM) and high glucose + quercetin $(100 \mu \mathrm{M})$ for $48 \mathrm{~h} ;{ }^{*} \mathrm{P}<0.05, \mathrm{n}=6$. (B) NO production in the culture medium of EPCs treated with high glucose (40 mM) and high glucose + quercetin $(100 \mu \mathrm{M})$ for $48 \mathrm{~h} ;{ }^{*} \mathrm{P}<0.05, \mathrm{n}=6$. (C) Intracellular superoxide dismutase levels in EPCs treated with high glucose (40 mM) and high glucose + quercetin $(100 \mu \mathrm{M})$ for $48 \mathrm{~h} ;{ }^{*} \mathrm{P}<0.05, \mathrm{n}=6$. 

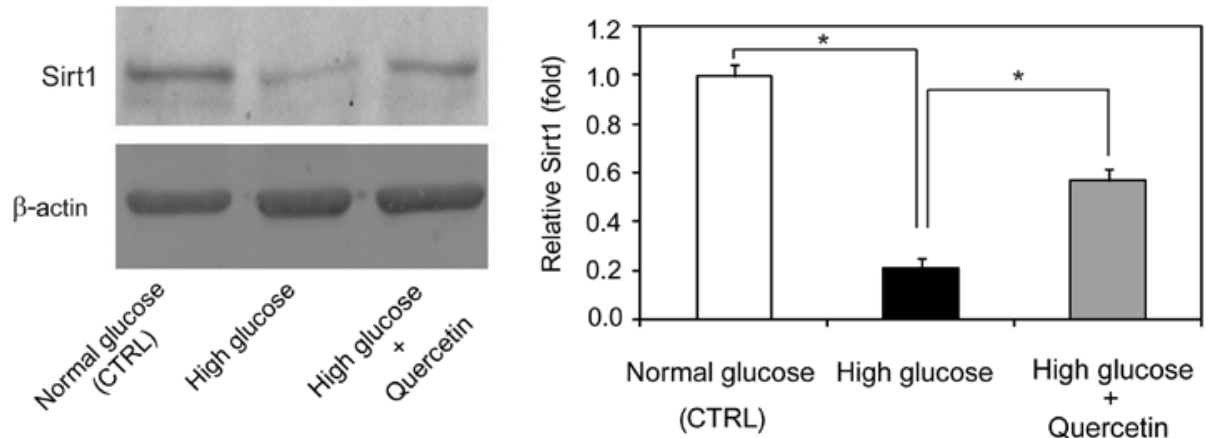

Figure 4. Effects of high glucose and quercetin on Sirt1 protein expression. Immunoblot analysis shows that high glucose (40 mM) decreases Sirt1 protein expression, which is partly prevented by quercetin $(100 \mu \mathrm{M})$. Endothelial progenitor cells (EPCs) were treated with high glucose and high glucose + quercetin for $48 \mathrm{~h} ;{ }^{*} \mathrm{P}<0.05, \mathrm{n}=5$.

A
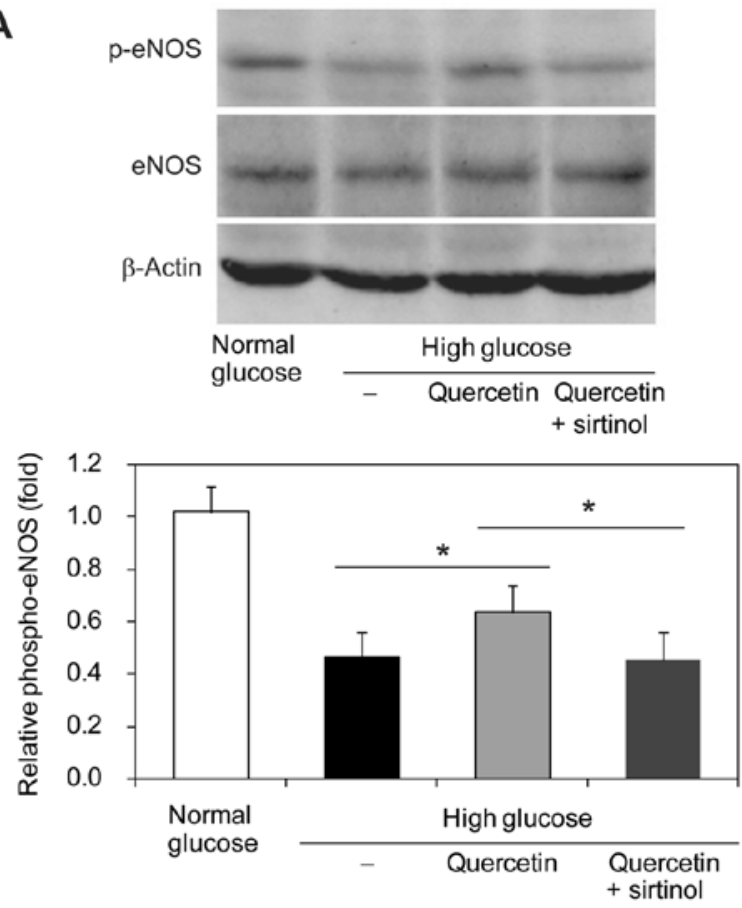

B

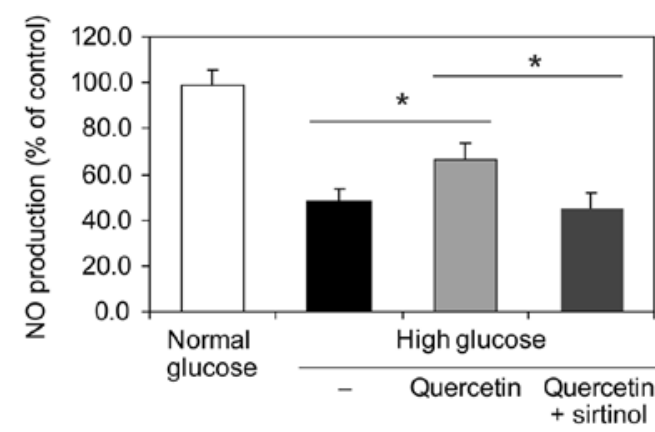

C

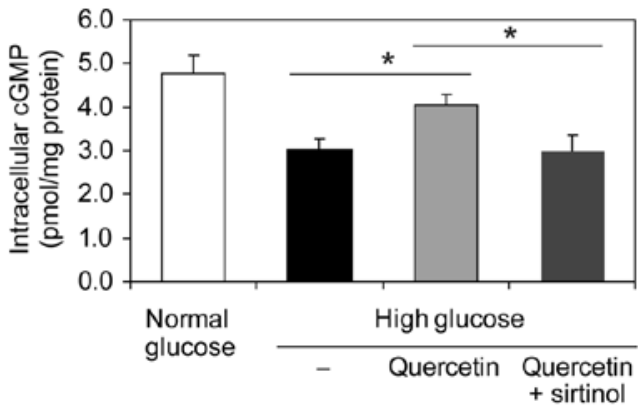

Figure 5. The protection of quercetin on endothelial nitric oxide synthase (eNOS) phosphorylation and nitric oxide (NO) production in endothelial progenitor cells (EPCs) is Sirt1-dependent. (A) Representative immunoblotting images and quantitative analysis of eNOS phosphorylation (phospho-eNOS/total-eNOS ratio) in EPCs treated with high glucose $(40 \mathrm{mM})$ or high glucose + quercetin $(100 \mu \mathrm{M})$ or high glucose + quercetin $(100 \mu \mathrm{M})+\operatorname{sirtinol}(40 \mu \mathrm{M})$ for $48 \mathrm{~h}$; ${ }^{*} \mathrm{P}<0.05$, $\mathrm{n}=6$. (B) Quantitative analysis of NO production in the medium of EPCs treated with high glucose $(40 \mathrm{mM})$ or high glucose + quercetin $(100 \mu \mathrm{M})$ or high glucose + quercetin $(100 \mu \mathrm{M})+\operatorname{sirtinol}(40 \mu \mathrm{M})$ for $48 \mathrm{~h}$; ${ }^{*} \mathrm{P}<0.05, \mathrm{n}=6$. (C) Quantitative analysis of intracellular cyclic guanosine monophosphate (cGMP) levels in EPCs treated with high glucose $(40 \mathrm{mM})$ or high glucose + quercetin $(100 \mu \mathrm{M})$ or high glucose + quercetin $(100 \mu \mathrm{M})+\operatorname{sirtinol}(40 \mu \mathrm{M})$ for $48 \mathrm{~h}$; $\mathrm{P}<0.05$, $\mathrm{n}=6$.

prevented the high glucose-induced inhibition of eNOS phosphorylation and NO production in EPCs.

Quercetin protects high glucose-induced inhibition of eNOS phosphorylation in a Sirt1-dependent manner in EPCs. Sirt1, a nicotinamide adenosine dinucleotide $\left(\mathrm{NAD}^{+}\right)$-dependent histone deacetylase, deacetylates eNOS, stimulating eNOS activity and increasing endothelial NO production (31). We studied the alteration of Sirt1 in EPCs following treatments of high glucose and quercetin. High glucose treatment decreased Sirt1 expression, whereas quercetin partly rescued the Sirt1 expression (Fig. 4). To elucidate whether this modulation on Sirt1 of quercetin is essential for the activation of eNOS, we used sirtinol, a specific inhibitor of Sirt1, to block Sirtl deacetylase activity. As shown in Fig. 5A, the sirtinol supplement completely blocked the rescuing effect of quercetin on eNOS phosphorylation. Furthermore, sirtinol blocked the increase of NO production (Fig. 5B) and intracellular cGMP (Fig. 5C) induced by quercetin treatment following high glucose stress.

\section{Discussion}

In the present study, we have demonstrated that quercetin protects against the high glucose-induced decrease of cell viability and migration in EPCs. Further experiments showed that quercetin decreased high glucose-induced oxidant 
stress, as evidenced by levels of ROS, MDA and superoxide dismutase. Quercetin prevented the high glucose-induced decrease of eNOS phosphorylation, NO production and intracellular cGMP level. Inhibition of Sirt1 by sirtinol also markedly blocked the rescuing effect of quercetin on eNOS phosphorylation, NO production and the intracellular cGMP level. These data suggest that quercetin protects against high glucose-induced damage in EPCs by restoring eNOS in a Sirt1-dependent manner.

In the present study, quercetin protects against high glucose-induced cell injury in EPCs. Previously, quercetin was reported to protect against high glucose-induced apoptosis in human umbilical vein ECs via its anti-oxidant effects (32). Bournival et al (33) and Shi et al (34) demonstrated a similar phenomenon in neuronal cells respectively. However, to the best of our knowledge, there is no report on the effects of quercetin on EPCs following high-glucose stress. Accumulating evidence suggests that oxidant stresscaused EPCs dysfunction is involved in the pathogenesis of diabetes (4-5). The number of EPCs obtained from type 1 diabetic patients in culture was $44 \%$ lower compared with age- and sex-matched control subjects (4). Similarly, EPCs from type 2 diabetic patients were reduced by $40 \%$ compared with healthy subjects (5). Additionally, conditioned media from patient EPCs were significantly reduced in their capacity to support endothelial tube formation in comparison to control EPCs (4). High glucose was shown to impair early and late EPCs by modifying NO-mediated mechanisms (35). Besides this mechanism, modulation of oxidant stress (6), apoptosis (7) and autophagy (36) also contribute to high glucose-induced cell damage. To the best of our knowledge, this is the first study on the protective effect of quercetin in high glucosechallenged EPCs, emphasizing the potential therapeutic value of quercetin in diabetic patients with trauma or surgery.

A novel mechanism underlying the protective effect of quercetin on EPCs under high glucose was identified in this study. To the best of our knowledge, this is the first study showing that quercetin prevented high glucose-induced inhibition of eNOS phosphorylation, NO production and intracellular cGMP in EPCs in a Sirt1-dependent manner. High glucose decreased eNOS and thereby NO production (35). NO donor although not antioxidants reversed the impairments, suggesting the role of NO-related mechanisms in high glucose-caused EPC downregulation (35). Moreover, uncoupling of eNOS in glucose-treated EPCs resulted in production of eNOS-mediated $\mathrm{O}^{2-}$ (37), which is detrimental to EPCs. In the present study, high glucose significantly decreased the phosphorylation of eNOS in EPCs and NO production. Of note, quercetin reversed this detrimental effect, suggesting that the improved NO signaling may account for the protective effect of quercetin in EPCs.

Of note, we found that quercetin upregulated Sirt1 in EPCs. Sirtl is a well-known key regulator of cell defenses, metabolism, aging and cell survival (38). High glucose has been reported to downregulate the number of EPCs by reducing Sirt1 expression levels and enzyme activity (39). Certain chemicals, such as stachydrine, may ameliorate high-glucose induced endothelial cell senescence and SIRT1 downregulation (40). The results of the present study demonstrate the enhancing effect on Sirt1 of quercetin in EPCs, which is in conconrdance with previous studies reporting that quercetin increases Sirt1 expression in brain and skeletal muscle (41-42). Moreover, we used sirtinol, an inhibitor of Sirt1, to investigate the effect of Sirt1 deacetylase activity inhibition on quercetin-induced eNOS upregulation and NO production. As expected, sirtinol efficiently inhibited the promoting effect of quercetin on eNOS upregulation and thereby NO production and cGMP concentration, suggesting that the beneficial effects of quercetin on EPCs is Sirt1-dependent.

In conclusion, to the best of our knowledge, we have demonstrated for the first time that quercetin protects against high glucose-induced damage by inducing Sirt1-dependent eNOS upregulation in EPCs. Thus, our results support the hypothesis that dietary supplement of quercetin may promote EPCs biological function and improve vascular repair, which is crucial for diabetic patients undergoing surgery or other invasive procedures.

\section{Acknowledgements}

This study was supported by the National Natural Science Foundation of China (no. 81272999).

\section{References}

1. Asahara T, Murohara T, Sullivan A, et al: Isolation of putative progenitor endothelial cells for angiogenesis. Science 275: 964-967, 1997.

2. Hristov M, Erl W and Weber PC: Endothelial progenitor cells: mobilization, differentiation, and homing. Arterioscler Thromb Vasc Biol 23: 1185-1189, 2003.

3. Hristov M and Weber C: Endothelial progenitor cells: characterization, pathophysiology, and possible clinical relevance. J Cell Mol Med 8: 498-508, 2004.

4. Loomans CJ, de Koning EJ, Staal FJ, et al: Endothelial progenitor cell dysfunction: a novel concept in the pathogenesis of vascular complications of type 1 diabetes. Diabetes 53: 195-199, 2004

5. Fadini GP, Miorin M, Facco M, et al: Circulating endothelial progenitor cells are reduced in peripheral vascular complications of type 2 diabetes mellitus. J Am Coll Cardiol 45: 1449-1457, 2005.

6. Sorrentino SA, Bahlmann FH, Besler C, et al: Oxidant stress impairs in vivo reendothelialization capacity of endothelial progenitor cells from patients with type 2 diabetes mellitus: restoration by the peroxisome proliferator-activated receptor-gamma agonist rosiglitazone. Circulation 116: 163-173, 2007.

7. Rosso A, Balsamo A, Gambino R, et al: p53 Mediates the accelerated onset of senescence of endothelial progenitor cells in diabetes. J Biol Chem 281: 4339-4347, 2006.

8. Hamed S, Brenner B and Roguin A: Nitric oxide: a key factor behind the dysfunctionality of endothelial progenitor cells in diabetes mellitus type-2. Cardiovasc Res 91: 9-15, 2011.

9. van Dam RM, Naidoo N and Landberg R: Dietary flavonoids and the development of type 2 diabetes and cardiovascular diseases: review of recent findings. Curr Opin Lipidol 24: 25-33, 2013.

10. Nicolle E, Souard F, Faure P and Boumendjel A: Flavonoids as promising lead compounds in type 2 diabetes mellitus: molecules of interest and structure-activity relationship. Curr Med Chem 18: 2661-2672, 2011.

11. Formica JV and Regelson W: Review of the biology of Quercetin and related bioflavonoids. Food Chem Toxicol 33: 1061-1080, 1995.

12. Kim JH, Kang MJ, Choi HN, Jeong SM, Lee YM and Kim JI: Quercetin attenuates fasting and postprandial hyperglycemia in animal models of diabetes mellitus. Nutr Res Pract 5: 107-111, 2011.

13. Margina D, Gradinaru D, Manda G, Neagoe I and Ilie M: Membranar effects exerted in vitro by polyphenols - quercetin, epigallocatechin gallate and curcumin - on HUVEC and Jurkat cells, relevant for diabetes mellitus. Food Chem Toxicol 61: 86-93, 2013. 
14. Mahmoud MF, Hassan NA, El Bassossy HM and Fahmy A: Quercetin protects against diabetes-induced exaggerated vasoconstriction in rats: effect on low grade inflammation. PloS One 8: e63784, 2013.

15. Zahedi M, Ghiasvand R, Feizi A, Asgari G and Darvish L: Does quercetin improve cardiovascular risk factors and inflammatory biomarkers in women with type 2 diabetes: a double-blind randomized controlled clinical trial. Int J Prev Med 4: 777-785, 2013.

16. Marrotte EJ, Chen DD, Hakim JS and Chen AF: Manganese superoxide dismutase expression in endothelial progenitor cells accelerates wound healing in diabetic mice. J Clin Invest 120 4207-4219, 2010

17. Wang P, Xu TY, Guan YF, Su DF, Fan GR and Miao CY: Perivascular adipose tissue-derived visfatin is a vascular smooth muscle cell growth factor: role of nicotinamide mononucleotide. Cardiovasc Res 81: 370-380, 2009.

18. Wang P, Guan YF, Du H, Zhai QW, Su DF and Miao CY: Induction of autophagy contributes to the neuroprotection of nicotinamide phosphoribosyltransferase in cerebral ischemia. Autophagy 8: 77-87, 2012.

19. Liu BQ, Du ZX, Zong ZH, et al: BAG3-dependent noncanonical autophagy induced by proteasome inhibition in HepG2 cells. Autophagy 9: 905-916, 2013.

20. Garg AD, Dudek AM, Ferreira GB, et al: ROS-induced autophagy in cancer cells assists in evasion from determinants of immunogenic cell death. Autophagy 9: 1292-1307, 2013.

21. Zhang XH, Lei H, Liu AJ, Zou YX, Shen FM and Su DF: Increased oxidative stress is responsible for severer cerebra infarction in stroke-prone spontaneously hypertensive rats. CNS Neurosci Ther 17: 590-598, 2011.

22. Schmeisser H, Fey SB, Horowitz J, et al: Type I interferons induce autophagy in certain human cancer cell lines. Autophagy 9: 683-696, 2013.

23. Luciani A, Villella VR, Esposito S, et al: Targeting autophagy as a novel strategy for facilitating the therapeutic action of potentiators on DeltaF508 cystic fibrosis transmembrane conductance regulator. Autophagy 8: 1657-1672, 2012.

24. Wang P, Zhang RY, Song J, et al: Loss of AMP-activated protein kinase-alpha2 impairs the insulin-sensitizing effect of calorie restriction in skeletal muscle. Diabetes 61: 1051-1061, 2012.

25. Wang P, Xu TY, Guan YF, et al: Nicotinamide phosphoribosyltransferase protects against ischemic stroke through SIRT1-dependent adenosine monophosphate-activated kinase pathway. Ann Neurol 69: 360-374, 2011

26. Oh JM, Choi EK, Carp RI and Kim YS: Oxidative stress impairs autophagic flux in prion protein-deficient hippocampal cells. Autophagy 8: 1448-1461,2012.

27. Zhang T, Li Y, Park KA, et al: Cucurbitacin induces autophagy through mitochondrial ROS production which counteracts to limit caspase-dependent apoptosis. Autophagy 8: 559-576, 2012.

28. Song YM, Song SO, Jung YK, et al: Dimethyl sulfoxide reduces hepatocellular lipid accumulation through autophagy induction. Autophagy 8: 1085-1097, 2012.
29. Zhao YD, Courtman DW, Deng Y, Kugathasan L, Zhang Q and Stewart DJ: Rescue of monocrotaline-induced pulmonary arterial hypertension using bone marrow-derived endothelial-like progenitor cells: efficacy of combined cell and eNOS gene therapy in established disease. Circ Res 96: 442-450, 2005.

30. Duda DG, Fukumura D and Jain RK: Role of eNOS in neovascularization: NO for endothelial progenitor cells. Trends Mol Med 10: 143-145, 2004.

31. Mattagajasingh I, Kim CS, Naqvi A, et al: SIRT1 promotes endothelium-dependent vascular relaxation by activating endothelial nitric oxide synthase. Proc Natl Acad Sci USA 104: 14855-14860, 2007.

32. Chao CL, Hou YC, Chao PD, Weng CS and Ho FM: The antioxidant effects of quercetin metabolites on the prevention of high glucose-induced apoptosis of human umbilical vein endothelial cells. Br J Nutr 101: 1165-1170, 2009.

33. Bournival J, Francoeur MA, Renaud J and Martinoli MG: Quercetin and sesamin protect neuronal PC12 cells from high-glucose-induced oxidation, nitrosative stress, and apoptosis. Rejuvenation Res 15: 322-333, 2012.

34. Shi Y, Liang XC, Zhang H, Wu QL, Qu L and Sun Q: Quercetin protects rat dorsal root ganglion neurons against high glucose-induced injury in vitro through $\mathrm{Nrf}-2 / \mathrm{HO}-1$ activation and NF-kappaB inhibition. Acta Pharmacol Sin 34: 1140-1148, 2013.

35. Chen YH, Lin SJ, Lin FY, et al: High glucose impairs early and late endothelial progenitor cells by modifying nitric oxide-related but not oxidative stress-mediated mechanisms. Diabetes 56: $1559-1568,2007$

36. Kobayashi S, Xu X, Chen K and Liang Q: Suppression of autophagy is protective in high glucose-induced cardiomyocyte injury. Autophagy 8: 577-592, 2012.

37. Thum T, Fraccarollo D, Schultheiss M, et al: Endothelial nitric oxide synthase uncoupling impairs endothelial progenitor cell mobilization and function in diabetes. Diabetes 56: 666-674, 2007.

38. Herranz D and Serrano M: SIRT1: recent lessons from mouse models. Nat Rev Cancer 10: 819-823, 2010.

39. Balestrieri ML, Rienzo M, Felice F, et al: High glucose downregulates endothelial progenitor cell number via SIRT1. Biochim Biophys Acta 1784: 936-945, 2008.

40. Servillo L, D'Onofrio N, Longobardi L, et al: Stachydrine ameliorates high-glucose induced endothelial cell senescence and SIRT1 downregulation. J Cell Biochem 114: 2522-2530, 2013.

41. Davis JM, Murphy EA, Carmichael MD and Davis B: Quercetin increases brain and muscle mitochondrial biogenesis and exercise tolerance. Am J Physiol Regul Integr Comp Physiol 296: R1071-R1077, 2009.

42. Casuso RA, Martinez-Lopez EJ, Nordsborg NB, et al: Oral quercetin supplementation hampers skeletal muscle adaptations in response to exercise training. Scand J Med Sci Sports: Oct 14, 2013 (E-pub ahead of print). 